\title{
La familia, también: integración y procedimientos de reunificación familiar para personas refugiadas en la Unión Europea
}

\author{
Leyre Benito Otazu*
}

\section{Resumen}

El grado de inclusión y protección que se podrá ofrecer a la población refugiada en los países de asilo de la Unión Europea depende de factores adicionales a los tradicionalmente considerados en relación al procedimiento de asilo o de los derechos en el asilo. Este artículo reivindica la centralidad de la familia como uno de dichos factores. Asimismo, examinando diversos procedimientos de reunificación familiar, tanto en el marco del Sistema Europeo Común de Asilo como al margen de él, reclama una coherencia entre los hallazgos de los estudios sobre integración de personas refugiadas y la tibia respuesta en el uso de las distintas vías para la reunificación familiar que se está dando en la actualidad.

\section{Palabras clave}

Reunificación familiar; Protección de refugiados; Integración de población refugiada; Interés superior del niño; Acceso a la información.

\section{-}

* Leyre BENITO

OTAZU, ha trabajado durante casi diez años con ACNUR en protección a personas refugiadas. Actualmente colabora en proyectos relacionados con asilo en España. leyrebenito@gmail.com.

Recibido: 2017-05-16

Aceptado:

2017-10-09

DOI: $10.15366 /$

relacionesinternacio nales2017.36.003

\section{TitLE}

The family too: Integration and family reunification procedures for refugees in the European Union

\begin{abstract}
The degree of inclusion and protection which the refugee population can be offered in countries of asylum within the European Union depends on factors above and beyond those traditionally considered, and which refer to asylum procedures or rights to asylum. This article puts forward the centrality of the family as one such factor. Likewise, through an examination of various family reunification procedures -both within the Common European Asylum System and outwith it-, it calls for coherence between the findings of studies on refugee integration and the present lukewarm application of family reunification.
\end{abstract}

\section{KeYWORDS}

Family reunification; Refugee protection; Refugee integration; Best interests of the child; Access to information 


\section{ntroducción}

Las llegadas de personas solicitando asilo en la Unión Europea (UE) experimentaron un incremento importante a partir de $2012^{1}$. Se trataba a menudo de jóvenes, con frecuencia hombres huyendo del servicio militar obligatorio en el entonces reciente conflicto en Siria. A ellos les siguieron más: familias enteras o bien las partes de las familias que aquellos habían tenido que dejar atrás. De tal modo que las personas que están detrás de aquellas primeras solicitudes de asilo terminan siendo varias veces más. Este mismo escenario de conflicto/ persecución-separación-reunión se repite en otros países en el mundo que acogen personas refugiadas ${ }^{2}$.

El texto que sigue examinará cómo concibe la $U^{3}{ }^{3}$ la reunificación familiar -incluyendo la de personas refugiadas-, cómo -o si- la posibilita, de qué otras formas puede esta producirse, y si con esto se responde de manera satisfactoria a garantizar el cumplimiento de un derecho.

En una primera parte tratamos, por un lado, sobre el reconocimiento de la reunificación familiar como derecho en el derecho internacional y europeo; por otro lado $-y$ por ceñirnos únicamente a la contribución de la reunificación familiar en la sociedad de acogida, sin ahondar en sus beneficios como mecanismo para evitar riesgos a la vida y seguridad de los familiares que se encuentran en lugares de origen o tránsito- sobre su reconocimiento como factor protector esencial una vez en la sociedad de acogida. En una segunda parte exponemos una serie de aspectos de que adolece la puesta en práctica de la reunificación familiar de personas refugiadas en la UE.

Antes, unas precisiones terminológicas. El texto se refiere a "personas refugiadas" de manera amplia, incluyendo a solicitantes de asilo, a refugiados reconocidos por la administración nacional, y a aquellos a los que se les ha reconocido otro tipo de protección internacional -como la llamada protección subsidiaria-.

Por "familia" hace referencia a la unidad de relación primaria con la que se identifica la persona. En este sentido, en algunos casos coincidirá con la relación de vínculo matrimonial y los hijos e hijas de tal unión. En muchos otros, será más amplia o diversa que esta, frecuentemente haciendo prevalecer vínculos de afecto y/o dependencia varios sobre los de parentesco genético. Esto se explica no solo por la multiplicidad de tradiciones socioculturales de las que proceden las personas refugiadas a las que nos estamos refiriendo, sino también por la propia evolución del concepto en los mismos lugares en los que se basaba la teoría que conceptualizaba la familia nuclear.

Con "reunificación familiar", el texto engloba las distintas vías por las que familias

1 Eurostat, "Asylum statistics": http://ec.europa.eu/eurostat/statistics-explained/index.php/Asylum_statistics [Consultado el 20 de septiembre de 2017].

2 Para una revisión detallada de categorizaciones en la literatura según los motivos conducentes a la separación, ver STAVER, Anne, Family Reunification: A Right for Forced Migrants? Working Paper 51, Refugee Studies Centre, Universidad de Oxford, Oxford, noviembre de 2008, p. 5: https://www.rsc.ox.ac.uk/files/publications/workingpaper-series/wp51-family-reunification-2008.pdf [Consultado el 18 de abril de 2017].

3 En el texto nos referimos a la UE como una totalidad, pero hay que precisar que no todos los Estados miembros han optado por obligarse por todos los instrumentos específicos en materia de asilo. 
anteriormente separadas pueden reunirse -así, reagrupaciones, apadrinamientos, etc.-.

\section{La reunificación familiar de personas refugiadas en la Unión Europea: reconocimiento como derecho y como factor esencial para la integración}

\subsection{La reunificación familiar de personas refugiadas en la Unión Europea como derecho}

La importancia del poder disfrutar de una vida sana en familia para el desarrollo de los individuos es reconocida por el cuerpo legislativo en derechos humanos. La Declaración Universal de los Derechos Humanos (DUDH) califica a la familia como "el elemento natural y fundamental de la sociedad"4, al tiempo que establece que "nadie será objeto de injerencias arbitrarias en [...] su familia [...]" y que, más bien, "toda persona tiene derecho a la protección de la ley contra tales injerencias o ataques" ${ }^{\prime \prime}$.

Los Pactos Internacionales de Derechos Humanos recogen la formulación de la DUDH: el Pacto Internacional de Derechos Civiles y Políticos lo hace en sus artículos veintitrés y diecisiete $^{6}$; el Pacto Internacional de Derechos Económicos, Sociales y Culturales lo hace en su artículo diez, que exige, además, a los estados que concedan a este elemento fundamental de la sociedad "la más amplia protección y asistencia posibles, especialmente para su constitución y mientras sea responsable del cuidado y la educación de los hijos a su cargo"7.

Con posterioridad, una serie de instrumentos legales han precisado tanto el derecho al respeto a la vida privada y familiar -incluyendo la formulación del principio del interés superior del niño- como su cara opuesta de la moneda: la obligación de los estados a actuar para restaurar una unidad familiar rota.

Así, el primer Protocolo adicional a la Cuarta Convención de Ginebra relativa a la protección de las personas civiles en tiempo de guerra (1949), de 1977, exige que los estados faciliten la reunificación familiar "de todas las formas posibles" durante un conflicto armado ${ }^{8}$.

Unos años más tarde, la Convención sobre los Derechos del Niño estipula que los niños no han de ser separados de sus padres contra su voluntad ${ }^{9}$, y exige a los estados parte que resuelvan "de forma positiva, humana y expedita"10 los casos de reunificación inter fronterizos.

\footnotetext{
4 Artículo 16.3, DUDH, 1948, en Oficina del Alto Comisionado de las Naciones Unidas para los Derechos Humanos (ACNUDH), Los principales tratados internacionales de derechos humanos, Naciones Unidas, Nueva York y Ginebra, 2006: http://www.ohchr.org/Documents/Publications/CoreTreatiessp.pdf [Consultado el 8 de septiembre de 2017].

5 Artículo 12, Ibídem.

6 Pacto Internacional de Derechos Civiles y Políticos, 1966, ibíd.

7 Pacto Internacional de Derechos Económicos, Sociales y Culturales, 1966, ibíd.

8 Artículo 74, Protocolo Adicional I de 1977, a la Cuarta Convención de Ginebra relativa a la protección de las personas civiles en tiempo de guerra (1949): http://www.acnur.org/t3/fileadmin/Documentos/BDL/2001/1387. pdf [Consultado el 8 de septiembre de 2017].

9 Artículo 9, Convención de los Derechos del Niño, 1989, en ACNUDH, op.cit.

${ }^{10}$ Artículo 10, Convención de los Derechos del Niño, Ibídem.
} 
Un comentario general del Comité de Derechos Humanos de Naciones Unidas de 1990, expone perfectamente la lógica argumental:

"el derecho a fundar una familia implica, en principio, la posibilidad de [...] convivir [...] la posibilidad de convivir implica la adopción de las medidas apropiadas, tanto al nivel interno como, según sea el caso, en cooperación con otros estados, para garantizar la unidad o reunificación de las familias, particularmente cuando sus miembros se encuentran separados por razones políticas, económicas o similares"11.

En el mismo año, la Carta Africana sobre los Derechos y el Bienestar del Niño, en su artículo veinticinco (2)(b), conmina a los estados parte a

"tomar todas las medidas necesarias para buscar y reunir a los niños con sus padres o parientes cuando la separación está causada por desplazamiento interno y externo derivado de conflictos armados o desastres naturales"12.

En el contexto europeo, como recoge de manera extensa un informe específico sobre el derecho de reunificación para personas refugiadas en la UE recientemente publicado ${ }^{13}$, el Convenio Europeo de Derechos Humanos recoge en su artículo ocho el derecho al respeto a la vida privada y familiar ${ }^{14}$. La Carta de los Derechos Fundamentales de la Unión Europea lo proclama en su artículo siete, y dedica un artículo separado, el veinticuatro, a los derechos del niño ${ }^{15}$. Adicionalmente, tanto el Convenio Europeo sobre el estatuto jurídico del trabajador migrante como la Carta Social Europea - revisada - ${ }^{16}$ incluyen reconocimiento y disposiciones en pro de la familia como unidad a la que hay que proteger.

Finalmente, desde 2003, una directiva europea aborda específicamente la reunificación familiar. En la sentencia por el caso Chakroun, en 2008, el Tribunal de Justicia de la Unión Europea (TJUE), interpreta esta directiva como reconociendo el derecho a la reunificación familiar. La sentencia considera que la Directiva ha de, no solo facilitar, sino promover la reunificación familiar, y garantizar que esta sea efectiva ${ }^{17}$. El Tribunal Europeo de Derechos Humanos (TEDH), por su parte, ha desarrollado una extensa jurisprudencia a partir de este instrumento, al ser confrontado con casos en que decisiones de política migratoria o penal de los estados - como expulsiones- impactaban en la vida familiar de personas residentes en territorio de los estados miembros o nacionales de los mismos. Según la jurisprudencia del

${ }^{11}$ Comité de Derechos Humanos de Naciones Unidas, Sesión 39, 1990, Comentario general 19 al artículo 23, párrafo 5: http://ccprcentre.org/page/view/general_comments/27791 [Consultado el 8 de septiembre de 2017].

${ }_{12}$ Artículo XXV (2)(b), Carta Africana sobre los Derechos y el Bienestar del Niño, 1990: http://www.acnur.org/t3/ fileadmin/Documentos/BDL/2010/8025.pdf [Consultado el 8 de septiembre de 2017].

${ }^{13}$ Council of Europe Commissioner for Human Rights, Realising the right to family reunification of refugees in Europe, Issue paper, Council of Europe, junio de 2017: http://www.coe.int/en/web/commissioner/familyreunification [Consultado el 8 de septiembre de 2017].

${ }^{14}$ Convenio Europeo de Derechos Humanos, 2010, p. 10: http://www.echr.coe.int/Documents/Convention_SPA. pdf [Consultado el 8 de septiembre de 2017]. El documento original data de 1970.

${ }^{15}$ Carta de los Derechos Fundamentales de la Unión Europea (actualizada), 2016: http://eur-lex.europa.eu/legalcontent/ES/TXT/?uri=celex:12016P/TXT [Consultado el 8 de septiembre de 2017]. El documento original data de 2000.

${ }_{16}^{16}$ Derecho/principio 16, Carta social europea (revisada), 1996, p. 7: https://rm.coe.int/168047e013 [Consultado el 8 de septiembre de 2017].

17 TJUE, caso Chakroun, caso C-578/08, de 4 de marzo de 2010: http://eur-lex.europa.eu/legal-content/EN/ TXT/?uri=CELEX\%3A62008C]0578 [Consultado el 8 de septiembre de 2017]. 
TEDH, la deportación de un miembro de una familia exige un análisis individualizado de los efectos sobre la vida familiar, y, en particular, sobre el contacto con los hijos ${ }^{18}$.

Entre un tribunal y otro, ha quedado establecido que toda decisión sobre reunificación familiar en la Unión Europea ha de estar justificada, ser necesaria y proporcional con el legítimo fin perseguido, y resultar efectiva en la práctica para que pueda darse la vida familiar y el derecho a la reunificación familiar para todo tipo de familias, sin discriminación.

¿Qué dice el derecho internacional de refugiados al respecto? Ni la Convención de Ginebra de 1951 sobre el Estatuto de los Refugiados (Convención de Ginebra) ni el Protocolo de Nueva York de 1967 abordan la reunificación familiar de personas en necesidad de protección internacional. A decir de un experto en derecho de refugiados, James Hathaway, los redactores de la Convención de Ginebra asumían que los casos de separación de familias por conflictos y persecución se resolverían vía estatuto derivado: una vez un miembro de la familia - "solicitante principal" - fuera reconocido como refugiado, el mismo estatuto se concedería al resto de la familia, una vez comprobada su relación ${ }^{19}$. No obstante, el Acta Final de la Conferencia de Plenipotenciarios que adoptó la Convención de Ginebra sí se refiere, en su Recomendación B, a la unidad familiar: esta recomendación exhorta a los estados a que "tomen las medidas necesarias para la protección de la familia del refugiado", por considerar que la unidad de la familia es "un derecho esencial"20.

Años más tarde, y con aplicación regional para América Latina, la Declaración de Cartagena sobre Refugiados afirma que la reunificación familiar debería ser la "base para el régimen de tratamiento humanitario en el país de asilo"21.

Por su parte, el Comité ejecutivo del Alto Comisionado de las Naciones Unidas para los Refugiados (ACNUR) 22 se ha pronunciado sobre la importancia de la reunificación familiar para las personas refugiadas en casi una decena de ocasiones desde $1975^{23}$. Si bien las conclusiones de dicho comité no dejan de ser recomendaciones, y, por tanto, carentes de fuerza vinculante, ACNUR es reconocida por los estados como voz relevante y referente en materia de asilo.

18 Por ejemplo, TEDH, caso Osman v. Dinamarca, 2011: https://ec.europa.eu/anti-trafficking/legislation-and-caselaw-case-law/case-osman-v-denmark_en [Consultado el 8 de septiembre de 2017].

19 HATHAWAY, James, The rights of refugees under international law, Cambridge University Press, Cambridge, p. 541.

${ }^{20}$ Recomendación B, Acta Final de la Conferencia de Plenipotenciarios.

${ }^{21}$ Coloquio Sobre la Protección Internacional de los Refugiados en América Central, México y Panamá: Problemas Jurídicos y Humanitarios, Conclusión III (13), Declaración de Cartagena sobre Refugiados, 1984: http://www. refworld.org.es/docid/50ac93722.html [Consultado el 8 de septiembre de 2017].

22 A 2017, compuesto por 101 Estados. Ver United Nations High Commissioner for Refugees (UNHCR), ExComm Plenary Session: http://www.unhcr.org/excom-plenary-sessions.html [Consultado el 29 de abril de 2017].

${ }^{23}$ Ver, por ejemplo: Conclusiones del Comité Ejecutivo número 1 (XXVI) 1975 (f); número 9 (XXVIII) 1977; número 24 (XXXII) 1981; número 84 (XLVIII) 1997; número 85 (XLIX) 1998 (u)-(X); número 88 (L) 1999. Disponibles a partir del Índice Temático Conclusiones del Comité Ejecutivo, junio de 2011: http://www.refworld. org.es/docid/57f76c1114.html [Consultado el 8 de septiembre de 2017]. 


\subsection{La reunificación familiar de personas refugiadas en la Unión Europea como factor esencial de integración}

Sin pretender sobrevalorar el concepto de familia, sabiendo que muchas familias se alejan del funcionamiento ideal, este apartado hace referencia a ella como unidad primaria en la que, en principio, la relación está tejida por amor, desarrollo, acompañamiento y respeto a los intereses de sus miembros.

Los estudios sobre integración de personas refugiadas en los países de asilo que existían hasta fechas muy recientes han sido principalmente temáticos -en torno a salud, empleo, relaciones intergeneracionales, trauma-, y con frecuencia han estado realizados por organizaciones no gubernamentales. Para la Unión Europea, en 2013, ACNUR observaba la "limitada investigación cuantitativa que incluyera detalle de indicadores de medición para refugiados" 24 . Aquellos estudios $-\mathrm{y} / \mathrm{o}$ indicadores- específicos sobre personas refugiadas tendían a centrarse en las áreas de más fácil medición de impacto: empleo, vivienda, educación, salud.

Con posterioridad, y dentro de una reflexión más amplia sobre qué constituye el bienestar, se están reivindicando las áreas "menos tangibles" de la integración, como son las relaciones sociales, la aceptación social o la percepción propia de la capacidad de desenvolverse en el entorno. Confluyen aquí varias líneas de análisis: las que privilegian el análisis desde abajo (bottom-up) y atribuyen el protagonismo a los sujetos -con plena capacidad de agencia y de influencia sobre las estructuras institucionales y normativas-, y la de las prácticas diarias (everyday practices) que nos habla de integración en el día a día (everyday integration), de espacios comunes de encuentro y de la prevalencia de lo local sobre lo nacional, del municipio frente al estado, en lo que atañe a la construcción de la integración. En esta línea son fundamentales tres investigaciones: por un lado, el reciente estudio específico con población refugiada reasentada en el Reino Unido, llevado a cabo por la Universidad de Sussex -Optimising refugee resettlement in the UK: a comparative analysis ${ }^{25}$-; por otro, los estudios sobre áreas con fuerte presencia de personas con experiencia migratoria reciente, ligados al Institute for Public Policy Research (IPPR) de Londres - Rethinking Integration ${ }^{26}$ y Shared ground: Strategies for living well together in an era of high immigration ${ }^{27}-$.

Específicamente dentro de este conglomerado relacional, el valor de las relaciones internas entre la familia, y el valor de las relaciones con el exterior facilitadas por la familia ha sido reconocido en varios estudios. No son estudios con o sobre población refugiada, pero los hallazgos son relevantes al caso igualmente.

${ }^{24}$ UNHCR, A New Beginning: Refugee Integration in Europe, septiembre de 2013', p. 18: http://www.unhcr. org/52403d389.html [Consultado el 19 de abril de 2017]. Traducción propia.

${ }^{25}$ Ver Universidad de Sussex, "Optimising refugee resettlement in the UK: a comparative analysis. Update: two years into the project", Global Studies, Sussex, 2015: https://www.sussex.ac.uk/webteam/gateway/file. php?name=research-briefing-refugee-resettlement-stage-2.pdf [Consultado el 22 de abril de 2017]. El estudio se desarrolló entre 2013 y 2017.

26 MCNEIL, Clare y CHERTI, Myriam, Rethinking Integration, IPPR, Londres, 2012: http://www.ippr.org/files/ images/media/files/publication/2012/10/rethinking-integration_Oct2012_9761.pdf [Consultado el 22 de abril de 2017].

27 SACHRAJDA, Alice; GRIFFITH, Phoebe, Shared ground: Strategies for living well together in an era of high immigration, IPPR, Londres, 2014: http://www.ippr.org/files/publications/pdf/shared-ground_Oct2014.pdf [Consultado el 22 de abril de 2017]. 
Richard Crisp y David Robinson compararon la familia con otras formas relacionales importantes, como los amigos o los vecinos ${ }^{28}$. La familia, encontraron, es fundamental, como factor de protección, en el desarrollo de la resiliencia para las nuevas generaciones, ofrece cuidado físico para los miembros más vulnerables - niños, niñas y adolescentes, personas mayores, otras con algún tipo de dependencia-y es fuente de apoyo emocional, de vinculación social y, a menudo, de sostén económico. Su investigación concluyó que la familia definitivamente ofrece una contribución práctica y diferencial. En aspectos como la calidad y la disponibilidad del apoyo emocional, o el servir de puerta para actividades sociales - visitas unos a otros o actividades en la comunidad más amplia, por ejemplo-, familia y amigos se encontraban a la par. Sin embargo, en cuanto a apoyo socio-económico, la familia -incluso la que se había trasladado de barrio- era el recurso de mayor confianza. Este apoyo se materializaba de maneras diversas: desde contribuciones monetarias - préstamos o donaciones- a contribuciones en especie -comida, ropa-; e incluso mediante la oferta de servicios del día a día: transporte - para ir a comprar, en emergencias, para ocio-, reparaciones, etc. Asimismo era fundamental para el cuidado de los niños: la familia se hace cargo, en contraposición con la asistencia más ad hoc de los vecinos - por ejemplo, echando una miradita a los niños en el parque-.

Sabemos también que, en el caso de las familias migrantes con hijos jóvenes, las oportunidades generadas por la crianza de estos tienen el potencial de ser un elemento importante de socialización para la unidad familiar a través de la interacción con otras familias -originarias del lugar o no- en espacios públicos o privados como parques, bibliotecas o centros deportivos, además de la escuela y las actividades alrededor de ella ${ }^{29}$.

Para las personas refugiadas entrevistadas para un estudio encargado por ACNUR y financiado por la UE en $2013^{30}$, la separación de su familia es uno de los tres aspectos fundamentales ${ }^{31}$ para su integración en el país de asilo. Si bien el contexto en estos momentos es netamente diferente, de modo particular a partir de 2015, esta preocupación sigue siendo central. Y es que, así como la presencia de la familia contribuye, su ausencia resta. La preocupación y ansiedad que esto puede generar en la familia en el país de asilo puede interferir gravemente con el desenvolvimiento cotidiano: no solo desde el punto de vista de la salud, sino también en todas las otras esferas, como la búsqueda de vivienda y empleo, la educación -incluyendo aprendizaje del idioma ${ }^{32}-$, o incluso la interrelación con otros -

${ }^{28}$ CRISP, Richard, y ROBINSON, David, Family, Friends and Neighbours: Social Relations and Support in Six Low Income Neighbourhoods, Research Paper No. 9, Centre for Regional Economic and Social Research, Universidad de Sheffield Hallam, septiembre de 2010: http://research.shu.ac.uk/cresr/living-through-change/documents/ RP9_FamilyFriendsandNeighbours.pdf [Consultado el 19 de abril de 2017]. Los autores realizaron 180 entrevistas en seis barrios de bajo nivel de renta en distintos puntos del Reino Unido.

${ }^{29}$ RYAN, Louise, Children, sociality and migrant networks: using visual data to map embedding in place, Conference proceedings, Migration and Families in Europe: National and Local Perspectives at a Time of Euroscepticism, Universidad de Manchester, 7-8 febrero de 2017. Como Ryan señala en su presentación, la interacción regular no deriva necesariamente en generación de capital social.

30 UNHCR, A New Beginning..., op.cit., p. 20. El estudio, llevado a cabo entre 2012 y 2013, obtuvo estas respuestas a partir de entrevistas semiestructuradas llevadas a cabo en Francia, Austria, Suecia e Irlanda.

${ }^{31}$ Los otros dos aspectos fueron la duración del procedimiento de asilo y la incertidumbre generada por el cambio de estatuto legal de solicitante de asilo a refugiado. Quedaban fuera de esta pregunta los aspectos identificados a nivel de políticas, como empleo, vivienda, educación, idioma.

32 Testimonios de profesores en Suecia (UNHCR, A New Beginning..., op.cit., p. 94) o testimonios de personas refugiadas hacen referencia al impacto que pueden tener materiales de estudio habituales, como estampas 
incluyendo la esfera pública-. Si la separación se produjo en circunstancias dramáticas, si no existen noticias del paradero de los seres queridos, o si estos quedaron en riesgo, el efecto puede ser aún mayor. Adicionalmente, desde el punto de vista de los miembros de la familia que no están en el país de asilo, la prolongación de esta situación aumenta su vulnerabilidad.

Los efectos que una separación prolongada puede tener, a la hora de reunirse, están igualmente documentados: la estructura y la relación intrafamiliar pueden verse dañados y ser fuentes de conflicto ${ }^{33}$.

El mantener o conseguir la unidad familiar para las personas refugiadas significaría, pues, desde esta óptica, la superación de uno de los hitos que ellas ven como esenciales y determinantes de su capacidad de desarrollo en la sociedad del país de asilo. Donde se produjo una ruptura de esta unidad familiar, el restablecimiento de la misma supondría un paso hacia la posibilidad de reconstruirse desde un escenario íntimo de normalidad, recuperando un factor de protección propio e intrínseco en el que encontrar apoyo psicológico, social y económico, al margen de los programas de atención y asistencia que puedan ofrecerse.

\section{La puesta en práctica de la reunificación familiar de personas refugiadas en la Unión Europea no está respondiendo a la necesidad ni, a menudo, a la legislación}

No existe un conjunto único de pasos con los que se completa una reunificación familiar para personas en necesidad de protección internacional, ni un conjunto único de actores involucrados. Esto se debe a la multiplicidad de circunstancias en las que se ha podido producir la separación, y en las que se encuentran las familias, los países de origen, los sistemas, instituciones y estructuras de los países de asilo y/o de reasentamiento, la presencia y capacidad de organizaciones intergubernamentales o no gubernamentales, la fortaleza de la llamada sociedad civil, entre otros.

\subsection{La puesta en práctica de la reunificación familiar de personas refugiadas en la Unión Europea a partir de las directivas comunitarias, el reglamento llamado de Dublín y el mecanismo de reubicación}

La Directiva sobre reunificación ${ }^{34}$ es el principal instrumento legislativo de la Unión Europea en relación a la reunificación familiar de personas refugiadas. Data de 2003, fecha en la que se empiezan a plasmar los primeros frutos de las negociaciones para la creación del llamado Sistema Europeo Común de Asilo (SECA). Si bien las personas que entran en su ámbito de aplicación son todos los "nacionales de un tercer país", dedica un capítulo específico a la reunificación familiar de personas refugiadas.

El SECA representa el marco normativo que se crea con el objetivo de armonizar los diferentes aspectos sobre sistemas de asilo en los estados miembros. Hasta que entraron en

familiares, conversaciones sobre excursiones y viajes, etc.

${ }_{33}$ Por ejemplo, ver Red Cross EU Office, European Council on Refugees and Exiles (ECRE), Disrupted Flight: The Realities of Separated Refugee Families in the EU, noviembre de 2014, p. 5: http://www.ecre.org/wp-content/ uploads/2016/07/ECRE-and-Red-Cross-EU-Disrupted-Flight_November-2014.pdf [Consultado el 18 de abril de 2017].

${ }^{34}$ Directiva 2003/86/CE del Consejo, de 22 de septiembre de 2003, sobre el derecho a la reagrupación familiar, DO L 251 de 3.10.2003, pp. 12-18: http://data.europa.eu/eli/dir/2003/86/oj [Consultado el 2 de septiembre de 2017]. 
vigor los instrumentos de la llamada primera fase, cada estado miembro se ceñía a sus leyes nacionales a la hora de decidir sobre casos de asilo. EI SECA pretende atacar a la disparidad que esto generaba en el seno de este espacio con fronteras comunes, $y$, al tiempo, pretende ofrecer una propuesta integral que aborde temas que se estaban poniendo en relación, como el control de fronteras.

El tronco del sistema lo conforman tres directivas - sobre procedimientos de asilo, sobre requisitos, sobre condiciones de acogida- y dos reglamentos - sobre la determinación del estado miembro responsable del examen de la solicitud de asilo, o Reglamento de Dublín; sobre Eurodac ${ }^{35}$ - En la actualidad, los cinco instrumentos han sido revisados ${ }^{36}, y$, para dos de ellos -Dublín y Eurodac, además del Reglamento que establecía la Oficina Europea de Apoyo al Asilo ${ }^{37}$-, las propuestas fruto de una nueva revisión ya están recibiendo comentarios expertos.

¿Cuáles son los puntos fundamentales en los que, desde el punto de vista de aplicación práctica del derecho comunitario referido a asilo, no se está haciendo corresponder la necesidad real y urgente de reunificación familiar con el proceso para su solicitud y consecución?

En primer lugar, según los instrumentos comunitarios, los derechos en relación a reunificación familiar no son los mismos para refugiados reconocidos que para beneficiarios de protección subsidiaria. Si bien en este texto, al optar por utilizar el término "refugiado" en sentido amplio no hemos evidenciado esta distinción, lo cierto es que tiene que tenerse en cuenta para interpretar las cifras de reunificaciones familiares concluidas en el período más reciente. La protección subsidiaria, en la UE, otorga protección internacional a aquellas personas para las que las autoridades nacionales en materia de asilo consideraron que no había motivos fundados de persecución individualizada contra ellos, pero que podrían sufrir daños graves en caso de ser devueltos a su país de origen.

A finales de 2016, los estados miembros de la UE habían reconocido a 263755

${ }^{35}$ La base de datos biométrica de la Unión Europea.

${ }^{36}$ Directiva 2013/32/UE del Parlamento Europeo y del Consejo, de 26 de junio de 2013, sobre procedimientos comunes para la concesión o la retirada de la protección internacional: http://data.europa.eu/eli/dir/2013/32/ oj; Directiva 2011/95/EU del Parlamento Europeo y del Consejo, de 13 de diciembre de 2011, por la que se establecen normas relativas a los requisitos para el reconocimiento de nacionales de terceros países o apátridas como beneficiarios de protección internacional, a un estatuto uniforme para los refugiados o para las personas con derecho a protección subsidiaria y al contenido de la protección concedida: http://data.europa.eu/eli/ dir/2011/95/oj; Directiva 2013/33/UE del Parlamento Europeo y del Consejo, de 26 de junio de 2013, por la que se aprueban normas para la acogida de los solicitantes de protección internacional: http://data.europa.eu/eli/ dir/2013/33/oj; Reglamento (UE) no 603/2013 del Parlamento Europeo y del Consejo, de 26 de junio de 2013, relativo a la creación del sistema "Eurodac" para la comparación de las impresiones dactilares para la aplicación efectiva del Reglamento (UE) no 604/2013, por el que se establecen los criterios y mecanismos de determinación del Estado miembro responsable del examen de una solicitud de protección internacional presentada en uno de los Estados miembros por un nacional de un tercer país o un apátrida, y a las solicitudes de comparación con los datos de Eurodac presentadas por los servicios de seguridad de los Estados miembros y Europol a efectos de aplicación de la ley, y por el que se modifica el Reglamento (UE) no 1077/2011, por el que se crea una Agencia europea para la gestión operativa de sistemas informáticos de gran magnitud en el espacio de libertad, seguridad y justicia: http://data.europa.eu/eli/reg/2013/603/oj [Consultados el 4 de octubre de 2017]. De modo especialmente relevante para este texto, para el Reglamento de Dublín revisado, ver Reglamento (UE) $n^{\circ}$ $604 / 2013$ del Parlamento Europeo y del Consejo, de 26 de junio de 2013, por el que se establecen los criterios y mecanismos de determinación del Estado miembro responsable del examen de una solicitud de protección internacional presentada en uno de los Estados miembros por un nacional de un tercer país o un apátrida (refundición): http://data.europa.eu/eli/reg/2013/604/oj [Consultado el 9 de septiembre de 2017].

${ }^{37}$ EASO, en sus siglas inglesas. 
personas como beneficiarias de protección subsidiaria, frente a 389670 como refugiadas $-y$ 56970 recibían protección humanitaria- ${ }^{38}$. Merece la pena revisar cómo ha evolucionado la situación, para el primer cuatrimestre de 2017, en algunos de los países que reciben el mayor número de solicitudes de asilo. Nos ceñiremos únicamente a refugiados versus beneficiarios de protección internacional, aunque sería interesante debatir también sobre la protección humanitaria -estatuto regulado exclusivamente por la legislación nacional-. Así, sobre el total de decisiones tomadas en primera instancia para el período, Alemania había reconocido un $25 \%$ de casos como refugiados, y un $22 \%$ como beneficiarios de protección subsidiaria; Italia, una cifra igual, del $10 \%$ de casos para ambos; Suecia, un $22 \%$ de casos como refugiados, y un $27 \%$ como beneficiarios de protección subsidiaria ${ }^{39}$.

Si bien los textos dan la opción de tratar en igualdad a personas reconocidas tanto como refugiadas como beneficiarios de protección subsidiaria, los estados están ejerciendo su discrecionalidad a la baja respecto a estos últimos. Por un lado, no se les está aplicando exención de los requisitos de alojamiento, seguro médico y recursos económicos ${ }^{40}$-exención obligatoria para personas refugiadas-. Por otro lado, un número de estados ha introducido un período de espera, después de su reconocimiento, antes de que puedan siquiera iniciar el trámite de la solicitud de reunificación. Así, desde junio de 2016, los beneficiarios en Austria de este tipo de protección han de esperar tres años para solicitar reunificación familiar ${ }^{41}$; en Alemania, dos años; en Suecia, solo podrán optar a la reunificación casos excepcionales entre aquellos que solicitaron asilo después del 24 de noviembre de $2015^{42}$. Adicionalmente, según la Directiva sobre reunificación, los beneficiarios de protección subsidiaria $-y$, de hecho, los de protección humanitaria-, no están exentos de que se les apliquen las llamadas medidas de integración -cívica- previa a la resolución de su solicitud de reunificación familiar ${ }^{43}$. Como señalan Sergio Carrera y Anja Wiesbrock, estas medidas parecen estar siendo usadas, en nombre de una apuesta por la integración, para prevenir la reunificación de las familias.

Ciertamente, las cifras anteriores pueden interpretarse en línea con un ambiente político donde se imponen las restricciones al asilo. No solo puede ser más conveniente en cuanto a recursos humanos $-\mathrm{y}$ financieros y temporales - el proceder con reconocimientos de protección subsidiaria: desde el punto de vista de los oficiales que examinan la solicitud es más sencillo argumentar el riesgo del retorno a un contexto de riesgo, que el indagar en los

${ }^{38}$ Eurostat, EU member states granted protection to more than 700000 asylum seekers in 2016, 70/2017, 26 de abril de 2017: http://www.europeanmigrationlaw.eu/documents/Eurostat-AsylumDecisions-2016.pdf [Consultado el 1 de mayo de 2017].

${ }^{39}$ Eurostat, First instance decisions by outcome, selected Member States, 1st quarter 2017: http://ec.europa. eu/eurostat/statistics-explained/index.php/File:First_instance_decisions_by_outcome,_selected_Member_ States,_1st_quarter_2017_update.PNG [Consultado el 12 de septiembre de 2017].

${ }^{40}$ Recogidos en el artículo 7.1 de la Directiva sobre reunificación.

${ }^{41} \mathrm{CZECH}$, Philip, "A right to family reunification for persons granted international protection? The Strasbourg caselaw, state sovereignty and EU harmonisation": http://eumigrationlawblog.eu/a-right-to-family-reunification-forpersons-under-international-protection-the-strasbourg-case-law-state-sovereignty-and-eu-harmonisation-2/ [Consultado el 19 de abril de 2017].

42 KOFMAN, Eleonore, Restrictions on migration of family members and implications for family life, Conference proceedings, Migration and Families in Europe: National and Local Perspectives at a Time of Euroscepticism, Universidad de Manchester, 7-8 de febrero de 2017.

${ }^{43}$ Artículo 7.2, Directiva sobre reunificación. Para mayor desarrollo sobre esta "integración cívica" ver CARRERA, Sergio y WIESBROCK, Anja, Civic integration of third-country nationals: nationalism versus europeanization in the common EU Immigration Policy, Centre for European Policy Studies (CEPS), octubre de 2009. 
hechos que rodearon la salida del solicitante - que puede o no ser gran comunicador, o siquiera estar centrado en lo que le preguntan en ese momento por razones muy válidas- o elaborar con base en los conocimientos sobre la situación política, económica y sociocultural del país de origen buscando elementos de persecución. Además, cada reconocimiento de protección subsidiaria va a suponer, frente al reconocimiento como refugiados, una menor probabilidad de que a estas personas les sigan otros miembros de su familia al estado en cuestión.

Este punto de la política de asilo de la UE está siendo denunciado como en violación del derecho a la no discriminación -incluyendo en función del estatuto legal- reconocido en el artículo catorce del Convenio Europeo de Derechos Humanos. Siendo la protección internacional una -el derecho internacional de refugiados no distingue varias categorías de protección internacional- resulta injustificada una doble escala de derechos.

En segundo lugar, la familia, atendiendo a la definición de sus miembros conforme a la Directiva sobre condiciones de acogida - art. 2(j) - y a la Directiva sobre requisitos - art. 2 (c) - , es nuclear y conformada con anterioridad a la huida del país de origen.

Algo más amplia es la versión de la Directiva sobre reunificación familiar, que se refiere a la familia existente no antes de la huida de país de origen sino antes o después ${ }^{44}$ de la entrada en el país del solicitante. Con todo, ni una Directiva ni otras reflejan la realidad de familias compuestas o recompuestas en la huida, el entendimiento cultural de la relación de compromiso ${ }^{45}$, las relaciones con personas sin vínculo genético, las necesidades de afecto y protección que puedan sentir adolescentes o jóvenes de más de 18 años.

De hecho, esta definición de miembros de la familia es uno de los ejemplos con los que Vincent Chetail ilustra la parcialidad con que las modificaciones introducidas a las directivas sobre condiciones de acogida, procedimientos de asilo y requisitos en lo que constituye la segunda fase del SECA cumplirían, en su opinión, con el derecho internacional ${ }^{46}$. Los estados miembros, recalca, siguen obligados por la norma internacional de proporcionar el tratamiento más favorable. Chetail afirma que esto mina la credibilidad del Sistema y que es contraproducente para el establecimiento de los ansiados estándares comunes.

El mismo riesgo de que se armonice la legislación pero en el nivel mínimo de los estándares existe cuando se trata de niños, niñas y adolescentes no acompañados quienes inician un procedimiento de reunificación familiar: los estados miembros tienen obligación de admitir a los ascendientes de primer grado de los niños, pero queda a su discreción el admitir al guardián legal u otro familiar si no viven los ascendientes de primer grado o no se les puede localizar ${ }^{47}$.

\footnotetext{
${ }^{44}$ Si bien el artículo 9.2 de la Directiva deja a discrecionalidad de los Estados miembros el que puedan limitar al "antes" la consideración de miembros de la familia.

${ }^{45}$ Según la Directiva sobre reunificación, salvo por aplicación de un criterio de flexibilidad en la interpretación, quedarían excluidos de la posibilidad de reunificación las personas unidas en compromiso - antes de la huida, en el tiempo en que la persona solicitante de reunificación reside en el país de asilo-.

${ }^{46}$ CHETAIL, Vincent, "The Common European Asylum System: bric-à-brac or system?" en CHETAIL, Vincent, et al. (eds.), Reforming the Common European Asylum System: the new European refugee law, Martinus Nijhoff, 2016, p. 20 de la versión original de febrero 2015.

${ }^{47}$ Artículo 10.3, Directiva sobre reunificación.
} 
De igual manera, aunque es positivo que se haya incorporado el criterio de dependencia como criterio sobre el que basar la solicitud de reunificación -lo hacen tanto la Directiva sobre

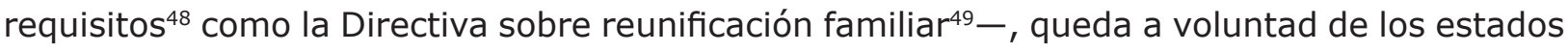
el admitir a personas fuera de las categorizadas como miembros de la familia. Ninguno de los dos instrumentos incluye una definición de dependencia ${ }^{50} \mathrm{y}$, como señalan Kate Jastram y Kathleen Newland, no existe una definición internacionalmente aceptada del término ${ }^{51}$.

En tercer lugar, existen una serie de requisitos como parte del procedimiento que se constituyen en barreras prácticas para conseguir concluir la reunificación. Aspectos temporales, documentales, económicos, u otros derivados de la situación específica del país de origen o asentamiento inicial de los familiares, no encuentran un acomodo adecuado en la legislación comunitaria. Los desarrollamos resumidamente a continuación.

Un factor de estrés importante es el temporal. Nos referimos aquí, por un lado, al período de tiempo que tarda el procedimiento de determinación del estatuto de refugiado -teniendo en cuenta que los estados miembros están haciendo depender el inicio de la solicitud de reunificación de la notificación del reconocimiento como refugiado, sin ofrecer la posibilidad de que los solicitantes de asilo inicien dicha reunificación-. Pese a que la Directiva sobre reunificación establece un plazo máximo de nueve meses para que el estado notifique la decisión sobre el caso, algunos estados están interpretando el comienzo del plazo no desde el inicio de la solicitud, sino desde que ellos confirman la recepción de esta. El lapso transcurrido entre uno y otra puede variar considerablemente según el contexto, como en el caso de las solicitudes presentadas en representaciones diplomáticas ${ }^{52}$.

También nos referimos, por otro lado, a la ventana de tiempo, posterior al reconocimiento, que la Directiva sobre reunificación establece como límite para cursar la solicitud de reunificación sin que los estados tengan la opción de exigir el cumplimiento de las condiciones materiales que se aplicarían en los casos de reunificación de personas de terceros países sin estatuto de refugiadas ${ }^{53}$ : alojamiento adecuado ("considerado normal"54) para la familia, seguro médico para sí mismos y sus familiares, y recursos estables y regulares para mantenerse todos ellos sin recurrir a los servicios sociales ${ }^{55}$. Esta era una discrecionalidad a la que no habían recurrido los estados hasta 2012, pero, a partir de entonces, tal y como apunta un informe recientemente publicado por el Consejo de Europa, la han ejercido países como Luxemburgo, Suecia, Hungría -esta última, a partir de mediados de $2016-{ }^{56}$. Estos

${ }^{48}$ Artículo 23.5, Directiva sobre requisitos.

${ }^{49}$ Artículo 10.2, Directiva sobre reunificación.

${ }^{50}$ Así, la Directiva sobre reunificación simplemente enuncia "si dependen del refugiado".

51 JASTRAM, Kate y NEWLAND, Kathleen, "Family Unity and Refugee Protection" en FELLER, Erika, TÜRK, Volker, y NICHOLSON, Frances, Refugee protection in international law: UNHCR's Global Consultations on International Protection, Cambridge University Press, junio de 2003, p. 585: http://www.refworld.org/docid/470a33be0. html [Consultado el 21 de septiembre de 2017].

52 Para el caso de España, ver el detalle del procedimiento en Red Cross EU/ECRE, Disrupted..., op.cit., p. 13.

${ }^{53}$ Artículo 12.1, Directiva sobre reunificación.

${ }^{54}$ Artículo 7.1.a, Directiva sobre reunificación.

${ }^{55}$ Artículo 7.1, Directiva sobre reunificación.

${ }^{56}$ Council of Europe Commissioner for Human Rights, Realising the right to family reunification of refugees in Europe, Issue paper, Council of Europe, June 2017, pp. 41-42: http://www.coe.int/en/web/commissioner/ 
tres meses se revelan escasos ante la realidad de muchas familias. La separación puede estar unida a una pérdida de contacto, con lo que es necesario iniciar procedimientos de búsqueda de familiares que implican a varios actores y se desarrollan con frecuencia en zonas en conflicto o riesgo de abusos. Asimismo, en relación con los requisitos de prueba documental, conseguir documentación de identidad o que demuestre filiación y parentesco en zonas donde la presencia de instituciones del estado está debilitada, es inexistente, requiere pagos excesivos o cuyo contacto puede entrañar un riesgo, puede constituir barrera insuperable para la familia en el país de origen o asentamiento inicial ${ }^{57}$. Para aquellos cuyos países 0 posición económica y social les permiten estar en posesión de esta documentación antes de la huida, estaríamos presuponiendo una planificación de la misma que no necesariamente puede darse. Finalmente, teniendo en cuenta que no existe presencia de consulados, embajadas u oficinas de procesamiento de visados en gran parte de los países donde se están dando situaciones de conflicto y persecución o en los de asentamiento inicial, el organizar el traslado -y a menudo, la estancia- de los familiares en la localidad donde han de realizar los trámites puede ser largo - a más de costoso-.

En estos momentos existe una propuesta de ampliación de este período de solicitud de tres a seis meses. No obstante, los aspectos anteriores requerirían que este más amplio margen estuviera acompañado de algún tipo de asistencia específica enfocada a las necesidades concretas de los solicitantes.

Un factor adicional es la exigencia de pruebas o evidencia documental del vínculo familiar, ya mencionado. En la formulación de la Directiva de reunificación: "una decisión de rechazo de la solicitud puede no estar únicamente basada en la ausencia de evidencia documental"58. Por tanto, puede que sí. De hecho, algunas solicitudes han sido rechazadas por falta de evidencia documental oficial -documento original apostillado, etc.- . Nuevamente, los países de origen de las personas refugiadas y la situación de las mismas son tremendamente diversos, pero, en un contexto de persecución, los documentos, si los hubo, pueden haber sido dejados atrás o haber desaparecido; y el solicitarlos al estado del que las personas huyeron podría ponerles en riesgo.

Como recoge el informe del Consejo de Europa de junio de 2017, particular dificultad en relación a la presentación de documentación están teniendo los hogares monoparentales y las familias con hijos no biológicos, así como aquellos casos en que se exigen pruebas de ADN -financiadas por los solicitantes- para establecer el parentesco59. Además, no se está dando ninguna flexibilidad a la hora de admitir nueva documentación una vez recibida la solicitud.

Finalmente, a lo largo del texto hemos mencionado factores económicos, y otros derivados de la situación específica del país de origen o asentamiento inicial de los familiares.

family-reunification [Consultado el 2 de septiembre de 2017].

${ }^{57}$ Como recoge el informe del Consejo de Europa de junio de 2017 (p. 43), particular dificultad en este sentido están teniendo los hogares monoparentales y las familias con hijos no biológicos.

${ }^{58}$ Artículo 11.2, Directiva sobre reunificación. Énfasis propio.

${ }^{59}$ Consejo de Europa, op.cit., p. 43: los Estados miembros solicitan autorizaciones de la pareja no presente cuando quizás no ha habido ni rastro de ella desde hacía mucho tiempo; en muchos países no se oficializa la adopción o el acogimiento de hijos no biológicos, y, sin embargo, se pide prueba documental de la validez de los mismos como parte del proceso en los Estados miembros-. 
No coincide con el hacer posible el ejercicio del derecho a la vida familiar el exigir de una familia de situación económica precaria el hacer frente a costes como minutas por traducción, desplazamientos a los puntos de procesamiento que correspondan - no necesariamente cercanos, si acaso llegan a estar en el mismo país-, etc. Estaríamos hablando de varios miles de euros por familia, en estimaciones de ACNUR para los casos de Bélgica y Noruega60. Algunas ONGs, de hecho, han desarrollado iniciativas de búsqueda de fondos específicamente para algunos de estos gastos de procesamiento de visados de familiares ${ }^{61}$.

En cuarto lugar, una vez concluido el proceso de reunificación, el ciclo de vida de las personas ha de ser tenido en cuenta para que el estatuto conferido garantice una protección estable a los familiares admitidos por esta vía. Algunos estados miembros han concedido un estatuto $-y$ correspondiente permiso de residencia, trabajo, etc.- dependiente de la continuidad de la unión del vínculo, lo cual genera problemas en casos de separación, divorcio, fallecimiento o violencia doméstica.

No podemos concluir este apartado sin notar que, para el caso de las personas nacionales de Siria residentes en territorio no europeo, y a fecha de 30 de abril de 2017, ACNUR no ha registrado ningún compromiso nacional para reunificaciones familiares ${ }^{62}$.

¿Qué papel desempeña el conocido como Reglamento de Dublín (III) en los procedimientos de reunificación familiar en la UE? El Reglamento 604/2013 establece "Ios criterios y mecanismos de determinación del estado miembro responsable del examen de una solicitud de protección internacional presentada en uno de los estados miembros por un nacional de un tercer país o un apátrida"63. Se refiere por tanto a aquellos casos en que los miembros de la familia están todos en territorio de la UE, mas en países diferentes. Atendiendo al enunciado del texto, se propone priorizar la unidad familiar. La práctica, no obstante, apunta al efecto contrario.

Así pues, en general, el estado responsable es aquel en el que se encuentre un familiar reconocido como refugiado o con una solicitud de asilo pendiente. Si la misma familia está repartida en varios estados miembros, la responsabilidad es de aquel donde se encuentra la mayoría de los familiares, o bien el solicitante de mayor edad.

En el caso de los niños, niñas y adolescentes no acompañados, y siempre que ello no contravenga el interés superior del niño, el estado responsable es aquel en el que se encuentre legalmente un miembro de la familia -incluyendo hermanos-. Lo mismo aplicaría en el caso de que el adolescente estuviera casado y su cónyuge residiera en la UE, mas sin

60 UNHCR, Comments on the proposal for a qualification regulation, diciembre de 2016, citada por Consejo de Europa, op.cit., pp. 43-44.

${ }^{61}$ Es el caso de la holandesa VluchtelingenWerk Nederland o la belga Un Visa, Une Vie, recogidos en el informe del Consejo de Europa mencionado, p. 44.

62 UNHCR, "Resettlement and Other Admission Pathways for Syrian Refugees", 30 de abril de 2017: http://www. refworld.org/docid/59786cf14.html [Consultado el 8 de septiembre de 2017].

${ }^{63}$ Reglamento (UE) N 604/2013 del Parlamento Europeo y del Consejo, de 26 de junio de 2013, por el que se establecen los criterios y mecanismos de determinación del Estado miembro responsable del examen de una solicitud de protección internacional presentada en uno de los Estados miembros por un nacional de un tercer país o un apátrida, DO L 180 de 29.6.2013, pp. 31-59: http://data.europa.eu/eli/reg/2013/604/oj [Consultado el 8 de septiembre de 2017]. 
estatuto legal. Respecto a la situación anterior, en que la familia del niño estuviera repartida en varios estados miembros, la decisión será tomada con base en la determinación del interés superior del niño. No obstante, informes sobre la materia reflejan una puesta en práctica en colusión con lo establecido. Así, fue en apelación únicamente que Holanda decidió no devolver a un niño a España como primer país de entrada. La madre del niño residía en Holanda, donde había conseguido la naturalización ${ }^{64}$. Por otro lado, no es posible una evaluación seria del interés superior del niño -que exige haber podido trabar un grado de confianza con él o ella, algo que no se logra con un contacto solamente- en estados donde, como es el caso de Grecia o Italia, están en marcha procedimientos acelerados para determinar la admisibilidad de los casos de asilo.

Aparte de lo mencionado, para que el mecanismo funcione es fundamental el acceso a la información: sin ella el ejercicio de derechos está limitado. Algunos solicitantes de protección internacional entrevistados por Action Aid Hellas -en Grecia - alegan no estar siendo debidamente informados de su opción de solicitar este procedimiento, ni de cómo avanza el mismo. Existe información escrita disponible en línea -en inglés- al respecto, en formato de folleto, pero puede resultar muy básica y es difícil encontrar la referencia a la posibilidad de apelar una decisión conforme al Reglamento de Dublín. El mensaje más repetido es que, si no procede Dublín, será Grecia quien examine la solicitud de asilo en su territorio65. Desconocemos cómo se está distribuyendo el folleto y si está disponible en otra lengua aparte de inglés. Lo cierto es que, en sus conclusiones en relación con la reforma del SECA, el Comité Económico y Social Europeo consideraba la información vía folleto ineficiente y recomendaba que se facilitara de manera oral, en la entrevista con los solicitantes ${ }^{66}$.

En segundo lugar, el funcionamiento no está siendo rápido: de nuevo refiriéndonos a Grecia, Action Aid Hellas informa haber entrevistado a personas que llevan más de ocho meses esperando una respuesta sobre su traslado a otro estado a través del Reglamento de Dublín III ${ }^{67}$. Las autoridades del país en que está el solicitante han de enviar la solicitud, y el potencial país de acogida ha de examinarla y enviar su respuesta, a más de coordinar la logística del traslado con la Organización Internacional de las Migraciones (OIM). Igualmente se prevén dificultades a la hora de poder apelar una decisión negativa sobre la responsabilidad del otro estado. Según establece el Reglamento, a falta de recursos legales privados, sería el

${ }^{64}$ RCEU/ECRE, Disrupted..., op.cit., p. 25.

65 Servicio de asilo griego, "Frequently Asked Questions and Answers": http://asylo.gov.gr/en/wp-content/ uploads/2016/07/Qandanswers.pdf, "Living with a close relative in another European country while your asylum claim is examined there (family reunification)": http://asylo.gov.gr/en/sp-content/uploads/2016/06/W001-4Family-Reunification-Poster.pdf, "Dublin III": http://asylo.gov.gr/en/?page_id=81 [Consultados el 20 de abril de 2017].

${ }^{66}$ Comité Económico y Social Europeo, "Opinión sobre el SECA", febrero de 2017: http://www.europeanmigrationlaw. eu/en/articles/news/european-economic-and-social-committee-opinion-common-european-asylum-systemceas [Consultado el 30 de abril de 2017].

${ }^{67}$ Action Aid, Separados. Los problemas de reubicación y reunificación familiar para los refugiados que llegan a Grecia, noviembre de 2016: http://www.alianzaporlasolidaridad.org/wp-content/uploads/Relocation_Report_ AA ENG_FINAL-LOW_page-by-page_08nov16 esp-002.pdf [Consultado el 19 de abril de 2017]. En declaraciones de una mujer afgana, de 31 años:

Estoy aquí con mi hija de nueve años, y mi marido está en Finlandia con mi otro hijo. Hemos solicitado la reunificación familiar y la última cita fue hace dos meses. Desde entonces no he sabido nada. Las autoridades de aquí me dijeron que tardan tres meses en examinar mis documentos en Finlandia y dos meses más para responder. No puedo entender por qué tardan tanto. Estoy pensando en irme ilegalmente. No sé lo peligroso que será, pero no sé qué hacer. ¿Y si la respuesta de Finlandia es negativa? ¿Nos enviarán de vuelta?" 
estado en el que se encuentra legalmente el solicitante el que debería facilitar esta respuesta, reenviando la solicitud de reunificación para que sea reconsiderada. La realidad de los países que en estos momentos están recibiendo el mayor número de solicitudes de reunificación familiar no permite gran optimismo.

El 4 de mayo de 2016, en el marco de la tercera fase del SECA, la Comisión Europea presentó la propuesta de reforma del Reglamento de Dublín: Dublín IV. La propuesta reafirma la primacía del derecho a la vida familiar y el cumplimiento del principio del interés superior del niño, bebiendo de la jurisprudencia hasta la fecha tanto del TEDH como de los tribunales nacionales, y superando en algunos aspectos las disposiciones de la Directiva sobre reunificación. Así, amplía la definición de miembros de la familia: incluye a los hermanos del solicitante, y a las relaciones familiares formadas con posterioridad a la huida del país de origen. Además, establece claramente la obligación de que personal especializado realice una evaluación del interés superior del niño antes de que ningún estado proceda a un traslado de un niño no acompañado68.

No obstante, con el nuevo texto, no se produce esta ampliación al después de la huida para los "dependientes", no se considera la dependencia más allá de por circunstancias de salud o trauma severo; no se fija un criterio común vinculante respecto a las parejas del mismo sexo, a aquellas en una relación estable pero sin vínculo matrimonial, o a los hijos adultos de la familia. Otros aspectos que recoge la propuesta y serían de dudosa compatibilidad con la legislación europea e internacional al respecto son, por ejemplo, la limitación de la representación legal para menores no acompañados al estado en que "deberían" estar presentes; o la imposibilidad de apelar la adjudicación de responsabilidad; la aplicación restrictiva del concepto de dependencia69.

En cuanto al mecanismo de reubicación que irrumpió en la realidad de la UE a finales de 2015, la reunificación de familias está contemplada como uno de los criterios que determinarían este traslado de solicitantes de asilo de nacionalidad siria, eritrea e iraquí - solo siria o eritrea desde junio de 2016, tras una leve disminución de la tasa media de reconocimiento en la UE para nacionales iraquíes- desde Grecia e Italia como países de entrada hasta los otros países en la UE donde residan sus familiares ${ }^{70}$ - Esto para las personas llegadas en un período de tiempo concreto que se fijó desde septiembre de 2015 hasta el 24 de marzo de $2016^{71}$. En estos casos, el caso de asilo se prevé sea examinado en el país de destino.

${ }^{68}$ Artículo 8.4 y considerando 20, propuesta de Reglamento del Parlamento Europeo y del Consejo estableciendo los criterios y mecanismos para determinar el Estado miembro responsable para el examen de la solicitud de protección internacional presentada en uno de los Estados miembros por un nacional de un país tercero o una persona apátrida (refundido), COM/2016/0270 final - 2016/0133 (COD): http://eur-lex.europa.eu/legalcontent/ES/TXT/PDF/?uri=CELEX:52016PC0270\&from=EN [Consultado el 8 de septiembre de 2017].

${ }^{69}$ Ver comentarios presentados en los meses transcurridos por diversos organismos especializados, como el Consejo de Europa, ECRE, ACNUR, EU-FRA.

70 Ver Servicio de asilo griego, "Frequently asked questions and answers on relocation": http://asylo.gov.gr/en/wpcontent/uploads/2015/11/RELOCATION_EN.pdf [Consultado el 30 de abril de 2017]. Este vínculo está enlazado a la página de la Oficina Europea de Apoyo al Asilo (EASO). Desde julio de 2017, a Eritrea y a Siria se le unieron Bahamas, Bahrein, Bután, Qatar, Emiratos Árabes Unidos, y Yemen como posibles países de procedencia de los susceptibles de ser trasladados.

${ }^{71}$ En esta fecha, la UE comenzó a aplicar una declaración firmada con Turquía que incluye disposiciones sobre devolución de solicitantes a fin de que su caso se decida en Turquía. 
¿Cuántos procesos de reunificación han podido darse a través de este mecanismo? De la información disponible, sabemos que, por un lado, frente a 14667 solicitudes entre enero y septiembre de 2016, solo 4637 se habían producido ${ }^{72}$. De entre ellas, no conocemos cuántas tomaron en cuenta la presencia de familiares en otros estados ${ }^{73}$. De lo que sí tenemos registro, a partir de las entrevistas realizadas en Grecia por Action Aid Hellas, es que, pese a que las personas pueden listar hasta ocho países de su preferencia, puede que sean reubicados a otro diferente, sin recibir mayor explicación sobre el motivo de la decisión ${ }^{74}$. A decir de Action Aid, "Ios solicitantes que no son parientes inmediatos pueden expresar su deseo de viajar juntos al mismo país, pero raramente se les concede" ${ }^{\prime \prime 7}$.

Contamos, por otro lado, con las cifras de niños, niñas y adolescentes (NNAs) no acompañados que han sido reubicados entre septiembre de 2015 y el 31 de agosto de 2017: desde Grecia han sido 309; desde Italia, 2076. Suponemos que estas cifras incluyen casos de traslados motivados por otras razones distintas de la reunificación familiar, lo que convierte en mínimos los casos de NNAs no acompañados reubicados para lograr la unidad familiar especialmente teniendo en cuenta que, en 2016, solo a Italia habían llegado más de 25000 NNAs no acompañados y separados ${ }^{77}$-.

Las cifras del total de personas reubicadas desde Grecia e Italia -independientemente del criterio-, a 4 de octubre de 2017, transcurrido el plazo comprometido, no permiten ser optimistas con esta vía para reunir familias: 29719 personas habían sido reubicadas sobre 106000 acordadas desde estos países de entrada ${ }^{78}$.

\subsection{La puesta en práctica de la reunificación familiar de personas refugiadas en la Unión Europea a través del reasentamiento y las "otras vías de admisión"79}

El reasentamiento ha sido considerado tradicionalmente por ACNUR como una solución duradera para la población refugiada. ACNUR facilita -a través de la OIM- el traslado de un individuo o familia ya reconocido como refugiado en un país de asentamiento inicial a un país distinto de este - a un "tercer país"- porque el estado de reasentamiento puede responder mejor a las necesidades de protección de la persona o familia. Al tiempo, contribuye a que los

\footnotetext{
72 Action Aid, Separados..., op.cit., p. 14.

${ }^{73}$ Uno de los criterios básicos para decidir sobre la responsabilidad de alojar y decidir sobre la solicitud de asilo - junto al conocimiento del idioma local o lazos culturales o sociales-. International Organization for Migration (IOM), "The EU relocation programme and IOM": http://eea.iom.int/index.php/what-we-do/eu-relocation [Consultado el 30 de abril de 2017].

${ }^{74}$ En ocasiones, la respuesta ha contenido referencias a razones de seguridad nacional y orden público. Action Aid, Separados..., op.cit., p. 17.

75 Action Aid, Separados..., Ibídem, p. 17.

${ }^{76}$ IOM, "IOM's activities in the EU relocation scheme": http://eea.iom.int/images/Download/Relocation\%20 Info\%20Sheet\%20-\%20August\%202017.pdf [Consultado el 2 de septiembre de 2017].

77 Notar que la cifra no solo incluye NNAs no acompañados, sino también separados - aquellos con un cuidador que no es quien de manera habitual realizaba esta función-. No conocemos cómo se divide esta cantidad entre unos y otros, pero lo exiguo de la cifra de reubicados desde el mismo país (20) permite suponer que es considerablemente más alta.

78 UNHCR, "UNHCR calls for the EU relocation scheme to continue": http://data2.unhcr.org/en/news/16558 [Consultado el 6 de octubre de 2017].

${ }^{79}$ Formulación usada por ACNUR y que engloba visados humanitarios, apadrinamiento privado, programas de becas académicas, mecanismos de contratación laboral, evacuaciones médicas (a más de reunificación familiar por vía nacional).
} 
países de asentamiento inicial no sufran - de la manera desproporcional en que lo hacen- las consecuencias de alojar en un lugar que les es desconocido, y de forma no planeada, a un número elevado de personas.

La reunificación familiar existe como criterio de reasentamiento ${ }^{80}$, pero, con el fin de que no termine restando "cupos"81 ya de por sí insuficientes para la necesidad, ACNUR se enfoca en fomentar que los estados utilicen los mecanismos nacionales propios para proceder con estas reunificaciones -simplificándolos, flexibilizándolos, acelerándolos-. Y es que, además, para que ACNUR pueda proceder con un caso de este tipo, al menos una de las personas que serán reasentadas ha de tener caso de asilo, y ser reconocida como refugiada bien por el estado del país de residencia bien, en su defecto, por ACNUR - a través del llamado reconocimiento bajo mandato-. De los casos presentados para reasentamiento entre enero y julio de 2017 , solo el $1 \%$ lo fueron bajo la categoría de reunificación familiar ${ }^{82}$. Siendo esta una cifra global, no ceñida a los países de la UE, da cuenta del escaso número de familias que están pudiendo ser reunidas por esta vía.

Respecto a las "otras vías de admisión", no nos estaríamos refiriendo ya a protección internacional concedida por los estados, sino a variados estatutos legales regidos por la legislación nacional. Son opciones que se adecúan a las necesidades de algunas personas, si bien pueden ofrecer menor garantía de la debida protección -en especial, contra la devolución- y pueden constituir una forma de invisibilizar éxodos de refugiados.

Una de las vías que podría ir en -relativo- crecimiento en la UE es el apadrinamiento privado. Está en marcha desde julio de 2016 en el Reino Unido ${ }^{83}$, en Italia, y Francia había anunciado un programa de este tipo para 2017-2018 ${ }^{84}$. Ha sido criticado por ser susceptible de quitar responsabilidad al estado de reasentamiento, pero lo cierto es que sigue siendo otra vía por la que las familias podrían unirse en el país donde ya se encuentran uno o varios de sus miembros. De hecho, la experiencia en Canadá muestra una preferencia de los padrinos por apadrinar a miembros de la familia extensa o conocidos de los refugiados previamente apadrinados ${ }^{85}$.

\section{Conclusión}

Existe amplio acuerdo en que la unidad de la familia constituye un derecho para el disfrute de la vida familiar, y en que la familia, en principio, es un elemento protector clave en la capacidad de integración de las personas refugiadas en el país de asilo.

${ }^{80}$ ACNUR, "Manual de Reasentamiento del ACNUR", julio 2011, pp. 300-316: http://www.refworld.org.es/ docid/52a0797c4.html [Consultado el 8 de septiembre de 2017].

${ }^{81}$ ACNUR negocia bilateralmente con un número de países - son unos pocos los que se ofrecen a participar-, de manera anual, cuántas familias van a poder acoger, y de qué países. Además de los criterios fijados por ACNUR y basados en necesidades de protección, cada país tiene unos criterios particulares.

82 UNHCR, "Global UNHCR submissions by category, January-July 2017": http://www.unhcr.org/resettlement-data. html [Consultado el 17 de septiembre de 2017].

83 IRIN, "So, your country isn't keen to resettle refugees. Are you?", 19 de junio de 2017: http://www.refworld.org/ docid/5948ef1c4.html [Consultado el 8 de septiembre de 2017].

${ }^{84}$ UNHCR, "Resettlement and Other Admission Pathways for Syrian Refugees", 30 de abril de 2017: http://www. refworld.org/docid/59786cf14.html [Consultado el 8 de septiembre de 2017].

85 HYNDMAN, Jennifer, et al., "Private refugee sponsorship in Canada" en Forced Migration Review, No 54, febrero 2017, p. 58. 
A lo largo de los años, la UE ha desarrollado una serie de medidas reguladoras de la reunión de las familias -si separadas- o el mantenimiento de su unidad. Esto incluye a las familias de las personas refugiadas, para las que existen disposiciones específicas, dentro del Sistema Europeo Común de Asilo. Con todo, un número importante de las mejoras en protección de la unidad de la familia han sido introducidas a partir de la acción de tribunales ante casos presentados por particulares y sociedad civil. Esta actitud renqueante, la armonización por criterios mínimos, la persistencia de ciertos dobles estándares en los derechos asociados a tipo de protección, las deficiencias en cuanto a garantías procedimentales -en acceso a información, en tiempos de resolución, por ejemplo-, son coincidentes con unas cifras oficiales inexistentes, en este último período reciente, para compromisos de los estados miembros ante ACNUR en lo que a reunificación familiar de refugiados sirios se refiere. Adicionalmente, otras vías que se formulan al margen del SECA y en clave nacional o privada, como pueden ser el reasentamiento o el apadrinamiento privado, no están preparadas $-\mathrm{y}$ parece que no se están preparando- para asumir el número de personas que se necesitaría para poder dar respuesta a la situación actual.

La reunificación familiar termina así ilustrando una tensión entre legislación y políticas; entre estándares supranacionales y discrecionalidad en la concreción nacional; entre una presión por la integración al interior y un discurso securitista en relación a las fronteras exteriores; entre solidaridad y temor a repercusiones electorales. Y una corta visión de futuro.

\section{Bibliografía}

Alto Comisionado de las Naciones Unidas para los Refugiados (ACNUR), "Manual de Reasentamiento del ACNUR, julio de 2011": http://www.refworld.org.es/docid/52a0797c4.html [Consultado el 8 de septiembre de 2017].

Action Aid, "Separados. Los problemas de reubicación y reunificación familiar para los refugiados que llegan a Grecia", noviembre de 2016: http://www.alianzaporlasolidaridad.org/wp-content/ uploads/Relocation_Report_AA_ENG_FINAL-LOW_page-by-page_08nov16_esp-002.pdf [Consultado el 19 de abril de 2017].

CARRERA, Sergio y WIESBROCK, Anja, Civic integration of third-country nationals: nationalism versus europeanization in the common EU Immigration Policy, Centre for European Policy Studies (CEPS), octubre de 2009.

CHETAIL, Vincent, "The Common European Asylum System: bric-à-brac or system?" en CHETAIL, Vincent, DE BRUYCKER, Philippe, y MAIANI, Francesco (eds.), Reforming the Common European Asylum System: the new European refugee law, Martinus Nijhoff, 2016.

Council of Europe Commissioner for Human Rights, Realising the right to family reunification of refugees in Europe, Issue paper, Council of Europe, June 2017: http://www.coe.int/en/web/commissioner/ family-reunification [Consultado el 8 de septiembre de 2017].

CRISP, Richard, y ROBINSON, David, Family, Friends and Neighbours: Social Relations and Support in Six Low Income Neighbourhoods, Research Paper No. 9, Centre for Regional Economic and Social Research, Universidad de Sheffield Hallam, septiembre de 2010: http://research.shu.ac.uk/ cresr/living-through-change/documents/RP9_FamilyFriendsandNeighbours.pdf [Consultado el 19 de abril de 2017].

European Council on Refugees and Exiles (ECRE) y European Legal Network on Asylum (ELENA), Information note on family reunification for beneficiaries of international protection in Europe, junio de 2016, pp. 36-37: http://www.ecre.org/wp-content/uploads/2016/07/ECRE-ELENAInformation-Note-on-Family-Reunification-for-Beneficiaries-of-International-Protection-inEurope_June-2016.pdf [Consultado el 20 de abril de 2017].

Eurostat, "Asylum statistics": http://ec.europa.eu/eurostat/statistics-explained/index.php/Asylum_ statistics [Consultado el 20 de septiembre de 2017].

Eurostat, "EU member states granted protection to more than 700000 asylum seekers in 2016", 70/2017, de 26 de abril de 2017: http://www.europeanmigrationlaw.eu/documents/EurostatAsylumDecisions-2016.pdf [Consultado el 1 de mayo de 2017]. 
Eurostat, "First instance decisions by outcome, selected Member States, 1st quarter 2017": http:// ec.europa.eu/eurostat/statistics-explained/index.php/File:First_instance_decisions_by_ outcome,_selected_Member_States,_1st_quarter_2017_update.PNG [Consultado el $1 \overline{2}$ de septiembre de 2017].

HYNDMAN, Jennifer, PAYNE, William y JIMÉNEZ, Shauna, "Private refugee sponsorship in Canada" en Forced Migration Review, No 54, febrero 2017, p. 58.

International Organization for Migration (IOM), "IOM's activities in the EU relocation scheme": http:// eea.iom.int/images/Download/Relocation\%20Info\%20Sheet\%20-\%20August\%202017.pdf [Consultado el 2 de septiembre de 2017].

International Organization for Migration (IOM), "The EU relocation programme and IOM": http://eea. iom.int/index.php/what-we-do/eu-relocation [Consultado el 30 de abril de 2017].

IRIN, "So, your country isn't keen to resettle refugees. Are you?", 19 de junio de 2017: http://www. refworld.org/docid/5948ef1c4.html [Consultado el 8 de septiembre de 2017].

JASTRAM, Kate y NEWLAND, Kathleen, "Family Unity and Refugee Protection" en FELLER, Erika, TÜRK, Volker, y NICHOLSON, Frances, Refugee protection in international law: UNHCR's Global Consultations on International Protection, Cambridge University Press, junio de 2003: http:// www.refworld.org/docid/470a33be0.html [Consultado el 18 de abril de 2017].

KOFMAN, Eleonore, Restrictions on migration of family members and implications for family life, Conference proceedings, Migration and Families in Europe: National and Local Perspectives at a Time of Euroscepticism, Universidad de Manchester, 7-8 febrero de 2017.

MCNEIL, Clare y CHERTI, Myriam, Rethinking Integration, Institute for Public Policy Research (IPPR), Londres, 2012: http://www.ippr.org/files/images/media/files/publication/2012/10/rethinkingintegration_Oct2012_9761.pdf [Consultado el 22 de abril de 2017].

Oxfam, the British Refugee Council, the British Red Cross y Amnesty International, "Together again: reuniting refugee families in safety", 2017: http://policy-practice.oxfam.org.uk/publications/ together-again-reuniting-refugee-families-in-safety--what-the-uk-can-do-620199 [Consultado el 22 de abril de 2017].

Red Cross EU Office, European Council on Refugees and Exiles (ECRE), "Disrupted Flight: The Realities of Separated Refugee Families in the EU", noviembre 2014: http://www.ecre.org/wp-content/ uploads/2016/07/ECRE-and-Red-Cross-EU-Disrupted-Flight_November-2014.pdf [Consultado el 18 de abril de 2017].

RYAN, Louise, Children, sociality and migrant networks: using visual data to map embedding in place, Conference proceedings, Migration and Families in Europe: National and Local Perspectives at a Time of Euroscepticism, Universidad de Manchester, 7-8 de febrero de 2017.

SACHRAJDA, Alice; GRIFFITH, Phoebe, Shared ground: Strategies for living well together in an era of high immigration, Institute for Public Policy Research (IPPR), Londres, 2014: http://www.ippr. org/files/publications/pdf/shared-ground_Oct2014.pdf [Consultado el 22 de abril de 2017].

Servicio de asilo griego, "Frequently asked questions and answers on relocation": http://asylo.gov.gr/ en/wp-content/uploads/2015/11/RELOCATION_EN.pdf [Consultado el 30 de abril de 2017].

Servicio de asilo griego, "Living with a close relative in another European country while your asylum claim is examined there (family reunification)": http://asylo.gov.gr/en/sp-content/uploads/2016/06/ W001-4-Family-Reunification-Poster.pdf [Consultado el 30 de abril de 2017].

Servicio de asilo griego, "Dublin III": http://asylo.gov.gr/en/?page_id=81 [Consultado el 20 de abril de 2017].

STAVER, Anne, Family Reunification: A Right for Forced Migrants?, Working Paper 51, Refugee Studies Centre, Universidad de Oxford, Oxford, noviembre de 2008: https://www.rsc.ox.ac.uk/files/ publications/working-paper-series/wp51-family-reunification-2008.pdf [Consultado el 18 de abril de 2017].

UN High Commissioner for Refugees (UNHCR), "UNHCR calls for the EU relocation scheme to continue": http://data2.unhcr.org/en/news/16558 [Consultado el 6 de octubre de 2017].

UN High Commissioner for Refugees (UNHCR), "Global UNHCR submissions by category, January-July 2017": http://www.unhcr.org/resettlement-data.html [Consultado el 17 de septiembre de 2017].

UN High Commissioner for Refugees (UNHCR), "Resettlement and Other Admission Pathways for Syrian Refugees", 30 de abril de 2017: http://www.refworld.org/docid/59786cf14.html [Consultado el 8 de septiembre de 2017].

UN High Commissioner for Refugees (UNHCR), "Proposal for a Regulation of the European Parliament and of the Council establishing a Union Resettlement Framework and amending Regulation (EU) No 516/2014 of the European Parliament and the Council UNHCR's Observations and Recommendations", noviembre de 2016: http://www.refworld.org/docid/5890b1d74.html [Consultado el 19 de abril de 2017].

UN High Commissioner for Refugees (UNHCR), "A New Beginning: Refugee Integration in Europe", septiembre de 2013: http://www.unhcr.org/52403d389.html [Consultado el 19 de abril de 
2017].

Universidad de Sussex, "Optimising refugee resettlement in the UK: a comparative analysis. Update: two years into the project", Global Studies, Sussex, 2015: http://www.sussex.ac.uk/migration/ refugeeresettlement [Consultado el 18 de abril de 2017]. 


\section{RELACIONES INTERNACIONALES}

Revista académica cuatrimestral de publicación electrónica Grupo de Estudios de Relaciones Internacionales (GERI) Universidad Autónoma de Madrid, España

www.relacionesinternacionales.info

ISSN 1699 - 3950

ff facebook.com/RelacionesInternacionales

twitter.com/RRInternacional 